\title{
Some new proposals for the classification of inherited myopathies
}

Dear Editor,

In a recent publication in the Journal of the Neurological Sciences, we highlighted the current difficulties in classifying the various forms of inherited myopathies [1]. These suggestions were based on our initial proposals for Charcot-Marie-Tooth disease (CMT) and other neurogenetic disorders) [2]. Recently, we conducted an internet survey to evaluate the interest of the scientific community: $65 \%$ of the people consulted considered that changes are needed and that our proposals constitute an improvement over the historical classification of CMT [3, 4]. There have also been recent attempts to solve such problems in other neurogenetic disorders such as hereditary ataxias [5]. We are aware that any new classification of neuromuscular disorders will need to be discussed in the neurological and genetic communities, but we have proposed simplifying the classification of inherited muscular disorders by using the same three successive steps: clinical phenotype, mode of inheritance, then the name of the causative gene (Fig. 1A) [1].

We would like to make an alternative proposal for a 'universal method' of classifying genetic disorders. In fact, it may be possible to completely redesign the naming of hereditary muscular disorders, based on the practical method of diagnosis in these diseases, which is the aim of our second proposal based on the main features (clinical, pathological, electrophysiological or radiological) employed in the diagnostic pathway of myopathy (whether dystrophic or not). Concerning step 1 (ie the first element appearing in the list of a database), it can be split into two successive modules (Fig. 1B). The first module of the 'step 1 ' corresponds to the 'generic term' of the entity, namely 'HM', for 'hereditary myopathy'. We have selected this term because of the large heterogeneity of muscular disorders: moreover, by using it, the term HM appears first in a database. For inherited neuropathies, the phenotypes are limited to three: CMT, dHMN (distal hereditary motor neuropathy) and HSAN (hereditary sensory and autonomic neuropathies); the same is true for other neurogenic diseases (SPG, etc.).

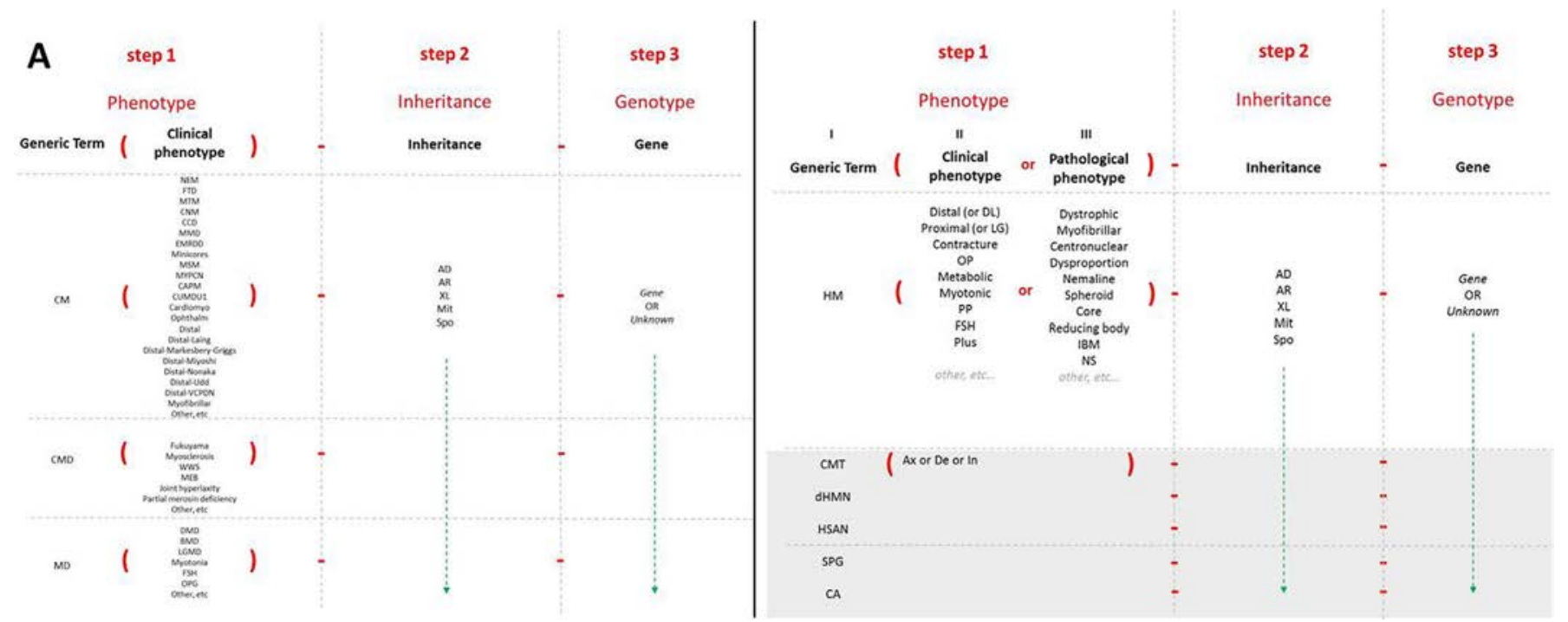

Fig. 1. A: first proposal for the classification of inherited myopathies (published in Journal of the Neurological Science) [1]. B: alternative proposal for the classification of the inherited myopathies (and other neurogenetic disorders, in grey box).

(AD: autosomal dominant; AR: autosomal recessive; Ax: axonal; BMD: Becker muscular dystrophy; CA: hereditary cerebellar ataxia; CAPM: Cap myopathy; Cardiomyo: cardiomyopathy; CCD: central core disease; CM: congenital myopathy; CMD: congenital muscular dystrophy; CNM: centronuclear myopathy; CUMDU1: congenital neuromuscular disease with uniform type 1 fiber; De: demyelinating; dHMN: distal hereditary motor neuropathy; DMD: Duchenne muscular dystrophy; EMARDD: early-onset myopathy, areflexia, respiratory distress and dysphagia; FSH: facioscapulohumeral dystrophy; FTD: fiber-type dysproportion HM: hereditary myopathy; HSAN: hereditary sensory and autonomic neuropathy; IBM: inclusion body myopathy; In: intermediate; LGMD: limb-girdle muscular dystrophy; MD: muscular dystrophy; MEB: muscle eye brain syndrome; Mit: mitochondrial inheritance; MMD: multiminicore disease; MSM: myosin storage myopathy; MTM: myotubular myopathy; MYPCN: Compton-North congenital myopathy; NEM: nemaline myopathy; NS: non-specific; OPG: oculopharyngeal; Ophthalm (or OP): ophthalmoplegia; PP: periodic paralysis; SPG: hereditary spastic paraplegia; VCPDN: vocal cord and pharyngeal dysfunction with distal myopathy; Spo: sporadic; WWS: Walker-Warburg syndrome; XL: X-linked). 
The two next parts of the 'step 1' include the 'clinical phenotype' (II: proximal, distal, joint contracture, etc.) and/or the 'pathological phenotype' (III: dystrophic, myofibrillar, etc.), into brackets (clinical and pathological parts being separated by a slash when both are used); moreover, it may also be possible to invert II and III. However, it is likely that molecular biological methods might soon supplant muscular biopsy in the determination of the pathological phenotype. Based on our experience, we propose a non-exhaustive list for each part (Fig. 1B): the type of myopathy affecting the patient is thus readily identified. It is also possible to group some clinical entities, such as MEB (Muscle-EyeBrain) or WWS (Walker-Warburg syndrome), in the same group that we may name 'plus'. In addition, other (not yet described) forms of HM can be added and it removes certain ambiguous terms such as 'LGMD' (because some myopathies with proximal involvement are not classified as LGMD when they might be) or 'congenital myopathy' (for which some authors also include late-onset forms of hereditary myopathy). In order to retain the term 'LGMD', we propose using 'LG' (= limb girdle), even if we think that the term 'proximal' is better: thus, muscular dystrophy with predominant proximal involvement (whether LGMD or not) would become 'HM(LG/dystrophic)-...'; for example, LGMD2L would become 'HM(LG/dystrophic)-AR-ANOS5'. In such a classification, most eponyms (which often complicate classifications) are discarded. Another advantage is also the ease of distinguishing dystrophic processes from non-dystrophic processes. For CMT, the 'clinical phenotype' (or 'electro-clinical phenotype') is limited to 'De' (demyelinating), 'Ax' (axonal) and 'In' (intermediate) which is a simplification; for the other neurogenetic diseases (such as spastic paraplegia), it is not necessary to put any term in brackets. For 'step 2' (mode of transmission), we retain the classical 'AD' (autosomal-dominant), 'AR' (autosomal-recessive), ' $\mathrm{X}$ ' (X-linked), 'mit' (mitochondrial transmission), and 'unknown' (if unknown inheritance). We do not feel that is necessary to specify whether it is a congenital form or not; however, ' $\mathrm{C}$ ' (in brackets) could be added directly after the inheritance (for example: ...-AD(C)...) if needed. Finally, 'step 3' remains the same, including the name of the gene or 'unknown' (if gene is unknown).

We are not alone in thinking that the current classifications of HM are too heterogeneous and complex, even for specialists. It is why we have now presented two proposals of classification which also simplify the terminology. These proposals will need to be discussed by other experts in myology, neuropathology and genetics. We also note that it may be possible and convenient to use the same structure of classification for any neuro-degenerative disorder [2].

\section{References}

[1] S. Mathis, M. Tazir, L. Magy, F. Duval, G. Le Masson, M. Duchesne, et al., History and current difficulties in classifying inherited myopathies and muscular dystrophies, J. Neurol. Sci. 384 (2018) 50-54.

[2] J.M. Vallat, C. Goizet, L. Magy, S. Mathis, Too many numbers and complexity: time to update the classifications of neurogenetic disorders? J. Med. Genet. 53 (2016) $647-650$.

[3] L. Magy, S. Mathis, G. Le Masson, C. Goizet, M. Tazir, J.M. Vallat, Updating the classification of inherited neuropathies: results of an international survey, Neurology 90 (2018) (e870-e6).

[4] C.J. Klein, Inherited neuropathy precision classification: what's in a name? Neurology 90 (2018) 445-446.

[5] M. Beaudin, C.J. Klein, G.A. Rouleau, N. Dupre, Systematic review of autosomal recessive ataxias and proposal for a classification, Cerebellum Ataxias 4 (2017) 3.

Stéphane Mathis ${ }^{\mathrm{a}}$, Meriem Tazir ${ }^{\mathrm{b}, \mathrm{f}}$, Guilhem Soléa ${ }^{\mathrm{a}}$ Laurent Magyc Gwendal Le Masson ${ }^{\mathrm{a}}$, Philippe Couratier ${ }^{\mathrm{c}}$, Karima Ghorab ${ }^{\mathrm{c}}$, Fanny Duval ${ }^{\mathrm{a}}$, Idoia Lacoste ${ }^{\mathrm{a}}$, Cyril Goizet ${ }^{\mathrm{d}, \mathrm{e}}$, Jean-Michel Vallat ${ }^{\mathrm{c},}$ ${ }^{a}$ Department of Neurology, Nerve-Muscle Unit (National reference center 'maladies neuromusculaires du grand sud-ouest'), University Hospital of Bordeaux (groupe hospitalier Pellegrin), Place Amélie Raba-Léon, 33000 Bordeaux, France.

${ }^{\mathrm{b}}$ Department of Neurology, University Hospital Mustapha Bacha, Algiers, Algeria

${ }^{\mathrm{c}}$ Department of Neurology (National reference center 'neuropathies périphériques rares'), University Hospital Dupuytren, 2 avenue Martin Luther King, 87042 Limoges, France

${ }^{\mathrm{d}}$ Department of Medical Genetics (National reference center 'maladies neuromusculaires du grand sud-ouest'), University Hospital of Bordeaux (groupe hospitalier Pellegrin), place Amélie Raba-Léon, 33076 Bordeaux,

France

${ }^{\mathrm{e}}$ MRGM Laboratory, INSERM U1211, University of Bordeaux, place Amélie Raba-Léon, 33076 Bordeaux, France

${ }^{\mathrm{f}}$ Laboratoire de Neurosciences, University of Algiers 1, Algiers, Algeria E-mail address: jean-michel.vallat@unilim.fr

* Corresponding author. 